\title{
Influencia de la familia sobre las conductas antisociales en adolescentes de Arequipa-Perú
}

\author{
Family influence on antisocial behavior in \\ adolescents from Arequipa, Peru
}

\author{
Renzo Rivera ${ }^{1}$ \\ Milagros Cahuana Cuentas ${ }^{2}$ \\ Universidad Católica San Pablo, Perú
}

\begin{abstract}
Resumen. El objetivo fue determinar la influencia de la familia sobre las conductas antisociales en adolescentes no institucionalizados. La muestra consistió en 929 alumnos de secundaria entre 13 y 17 años de edad. Se aplicó una batería de instrumentos sobre datos sociodemográficos, conductas antisociales y funcionamiento familiar: relación, satisfacción, cohesión, adaptabilidad y comunicación familiar. Se analizaron las variables por medio de modelos de ecuaciones estructurales diferenciados por sexo. Los resultados mostraron que el funcionamiento familiar, en ambos sexos, y el número de hermanos, en los varones, son factores protectores frente a las conductas antisociales. Los factores de riesgo son: maltrato infantil y violencia entre los padres, en las mujeres; además del consumo de alcohol en los padres, para ambos sexos.
\end{abstract}

Palabras clave. Funcionamiento familiar, conducta antisocial, adolescentes no institucionalizados, maltrato infantil, modelo ecológico.

Abstract. The aim was to determine the influence of the family on antisocial behavior in non-institutionalized adolescents. The sample consisted of 929 high school students, between 14 and 17 years old. In the process, a battery of test about social-demographic data, antisocial behavior and family functioning: relationship, satisfaction, cohesion, adaptability and family communication was applied. We analyzed variables using structural equation models differentiated by sex. The results indicated that family functioning in both sexes and the number of siblings in males are considerable factors when discussing antisocial behavior. The risk factors are: child abuse and violence between parents in females, besides consumption of alcohol by parents in both sexes.

Keywords. Family functioning, antisocial behavior, non-institutionalized adolescents, child abuse, ecological model.

\footnotetext{
${ }^{1}$ Renzo Rivera. Miembro del Grupo de Investigación Educativa Schola e investigador del Instituto para el Matrimonio y la Familia de la Universidad Católica San Pablo. Dirección postal: Quinta Vivanco s/n, Urb. Campiña Paisajista, Arequipa, Perú. C.P. 04000. Email: crrivera@ucsp.edu.pe

${ }^{2}$ Milagros Cahuana Cuentas. Miembro del Grupo de Investigación Educativa Schola e investigadora del Instituto para el Matrimonio y la Familia de la Universidad Católica San Pablo, Perú. E-mail: micahuana@ucsp.edu.pe
}

\section{(c) (i) (9)}




\section{Introdución}

La adolescencia es una etapa de transición de la niñez a la adultez, que conlleva múltiples cambios físicos, cognoscitivos y psicosociales (Papalia, Wendkos, \& Duskin, 2005). Cuando los cambios antes mencionados se combinan con problemas familiares y/o contextos ambientales desfavorables, estos pueden influir en que los adolescentes presenten diversos problemas comportamentales, entre ellos conductas antisociales y, como consecuencia, lleguen a involucrarse en actos delictivos (Quiroz del Valle et al., 2007; Romero, Luengo \& Gómez-Fragüela, 2000).

El concepto de conducta antisocial hace referencia, básicamente, a una serie de actos que infringen las reglas o normas sociales y/o sean una acción contra los demás, independientemente de su gravedad o de las consecuencias que a nivel jurídico puedan acarrear (Peña \& Graña, 2006). Dichas conductas pueden variar en su gravedad, cronicidad y frecuencia de presentación, e incluyen una diversa gama de comportamientos que están en relación con la edad y comprenden actos legalmente definidos como delictivos; ya sea hurtos, vandalismo, piromanía o asesinatos; hasta una variedad de conductas no delictivas como las acciones agresivas, mentiras, absentismo escolar, falsificación de notas, fumar, beber alcohol, fugarse de casa y otras más (Garaigordobil, 2005; Kazdin, 1993; López \& da Costa, 2008; Villareal, 2009).

Las conductas antisociales presentan diversas consecuencias; primero, para el adolescente agresor que a corto plazo le puede significar una pérdida de amistades, e incluso ser suspendido o expulsado del colegio (Gaeta \& Galvanovskis, 2011); mientras que, a largo plazo, una parte de los niños y adolescentes con conductas antisociales llegados a la adultez, suelen continuar con conductas de tipo criminal, alcoholismo, afectación psiquiátrica, problemas en el trabajo y problemas familiares (Kazdin, 1993). Es necesario mencionar que no todos los adolescentes persisten con este comportamiento al llegar a ser adultos, dependerá de la trayectoria de vida con la que surja la conducta antisocial (Morales, 2008). En muchos países latinoamericanos, los jóvenes cometen delitos violentos y mueren por efecto de ellos a edades cada vez más tempranas (CEPAL, 2008). También se encuentran las consecuencias personales y económicas para las víctimas de dichos actos que pueden incluir a los hermanos, compañeros de clase, padres, docentes y personas desconocidas (Kazdin, 1993).

En Perú, la delincuencia se ha incrementado en los últimos años (Morales, 2013). Se ha calculado que por cada incidente, una víctima de pandillaje gasta en promedio 873.3 soles en el tratamiento de sus heridas y unos 416.9 soles en su rehabilitación, sin tener en cuenta el monto de los bienes que puede haber perdido. Anualmente, este problema genera un costo a la población peruana de más de 108 millones de soles; mientras que el Estado, en el año 2008, llegó a asumir un gasto de alrededor 71 millones de soles para combatir las bandas organizadas y el pandillaje, monto que crece cada año (Mirella, 2011).

A lo largo de la literatura se han venido investigando diferentes teorías que expliquen la génesis de la violencia y las conductas antisociales. Terrie Moffitt propone una teoría taxonómica de la conducta antisocial, dividiendo esta en dos tipos: la que persiste en el curso de la vida y la limitada a la adolescencia (Moffitt, 1993, como se citó en Morales, 2013). La diferencia entre ambos tipos de conducta antisocial se encuentra en su trayectoria de desarrollo (Herrera \& Morales, 2005). La conducta antisocial persistente en el curso de la vida se origina en la niñez, como consecuencia genética y biológica; el adolescente antisocial presenta déficiten su comprensión, atención y concentración además de otros problemas neurológicos (Nagin, Farrington \& Moffitt, 1995).

El segundo tipo de conducta antisocial es aquella limitada a la adolescencia; esta se caracteriza por la ausencia de problemas en el desarrollo neurológico del niño, por lo que su origen se remontaría a una explicación ambiental más que de tipo individual (Morales, 2008). Al respecto, el modelo ecológico propuesto por Bronfenbrenner (1987) muestra una base explicativa ambiental respecto a la conducta humana. Ha sido ampliamente utilizado como base en la explicación holística sobre las causas 
y consecuencias del maltrato infantil, conductas antisociales y demás temas asociados con la violencia. A nivel latinoamericano, Sanabria y Uribe (2009) además de Martínez, Robles, Utria y Amar (2014), realizaron investigaciones de corte ecológico sobre las manifestaciones y la legitimización de la conducta antisocial en adolescentes colombianos. Por otro lado, la importancia de las variables familiares en la conducta antisocial aplicando el modelo de Bronfennbrenner, en los países de Chile y México, respectivamente (Frías, Rodriguez \& Gaxiola, 2003; Florenzano, et al., 2009; Martínez, Robles, Utria, \& Amar, 2014; Sanabria \& Uribe, 2010). En el Perú, concretamente en Arequipa, Carpio de La Torre y Alay (2012) utilizaron el modelo ecológico como base explicativa de la conducta antisocial en adolescentes institucionalizados.

El modelo de Bronfennbrenner concibe el ambiente ecológico como un conjunto de estructuras seriadas y concéntricas en diferentes niveles, las cuales se contienen entre sí y que afectan directa o indirectamente el desarrollo de las personas (Frías, Rodriguez \& Gaxiola, 2003; Martínez, Robles, Utria, \& Amar, 2014). El ambiente ecológico se divide en cuatro niveles: el microsistema, nivel más inmediato en el que se desarrolla la persona, comprende a la familia, y las dinámicas que al interior de ella suceden; el mesosistema comprende las interrelaciones de dos o más entornos en los que se encuentra inmersa la persona (parientes, vecinos, amigos); el exosistema lo comprenden contextos más próximos al sistema familiar, el cual incluye a la escuela, iglesia, instituciones recreativas y organismos de control social; y el macrosistema, conformado por la cultura, creencias y actitudes de cada persona e individuos de su sociedad. Estos niveles dependen uno del otro y, por tanto, se requiere de una participación conjunta de los diferentes contextos y de una comunicación entre ellos (Bronfenbrenner, 1987).

La familia asume un peso importante dentro de este modelo ya que se halla íntimamente relacionada con el desarrollo del adolescente dentro del microsistema y el mesosistema. Belsky (2005) sostiene que la familia, como microsistema, es la principal influencia en cuanto al desarrollo psicológico de niños y adolescentes, esto porque la sensitividad de los padres en la crianza promueve la sensación de seguridad en los hijos; mientras que el control coercitivo o altos niveles de conflicto entre los padres se asocian a conductas antisociales.

En las investigaciones empíricas sobre la influencia de la familia en las conductas antisociales, los principales factores son divididos en dos grupos: los de tipo estructural y aquellos enfocados en el funcionamiento familiar (Antolín \& Oliva, 2009; Quiroz del Valle, et al., 2007). En el primer grupo encontramos: estructura familiar desintegrada por separaciones o divorcios y las familias uniparentales (Torrente \& Rodríguez, 2004), número de hermanos (Antolín, 2011; Torrente \& Rodríguez, 2004), ausencia del cuidador, insuficiencia de ingresos (Cuevas del Real, 2004; Quiroz del Valle et al., 2007; Rodríguez \& Torrente, 2003).

Los factores del funcionamiento familiar relacionados con las conductas antisociales son: un clima familiar caracterizado por una pobre cohesión y falta de normas claras (Matalinares et al., 2010), patrones de comunicación poco fluidos o comunicación rígida (Estévez, et al., 2007), una pobre satisfacción familiar (Araujo, 2005; Ponce, 2003), presencia de violencia transgeneracional, que los hijos presencien violencia entre sus padres o experimenten castigos físicos (Benavides \& León, 2013; Gracia, Fuentes, \& García, 2010; Martínez, et al., 2014; Noroño, Cruz, Cadalso, \& Fernández, 2002), estilos educativos erróneos de los padres como la legitimización del castigo o maltrato infantil (Cuevas del Real, 2004; Gaxiola \& Frías, 2008; Larrain, 2008; Quiróz del Valle et al.; Rodriguez \& Torrente, 2003) y que los padres consuman drogas o alcohol (Frías, Rodríguez, \& Gaxiola, 2003).

Asimismo, diversos estudios han indicado que las conductas antisociales difieren entre varones y mujeres en su forma, frecuencia y variabilidad de presentación (Jiménez, Estévez, \& Murgui, 2014; Martínez \& Gras, 2007; Quiroz del Valle, et al., 2007; Secretaría Nacional de la Juventud - MINEDU, 2013, Herrera \& Morales, 2005). En nuestra ciudad se encontró que las conductas antisociales en la escuela ligadas al bullying son mayores en los varones, los que tienden más a la agresión física y la intimidación; mientras que las mujeres agreden de 
forma verbal o a través del rechazo social (Bellido et al., 2013). Incluso, se halló que el efecto de diversos factores sobre la conducta antisocial es diferente entre varones y mujeres (Sobral, et al., 2000).

Si bien es cierto que el modelo teórico mencionado anteriormente (Belsky, 2005; Bronfenbrenner, 1987) apoya la importancia de la familia en la comprensión de las conductas antisociales en los adolescentes, es escasa la cantidad de estudios con una perspectiva integradora en el Perú. Las investigaciones referidas al tema se han hecho mayormente en muestras de adolescentes institucionalizados (Herrera \& Morales, 2005; Morales, 2008, 2013; Carpio de La Torre \& Alay, 2012) o el diseño de investigación era bivariado, analizando las relaciones entre variables aisladas y dificultando, de esta forma, la obtención de datos que nos brinden una visión global del papel que la familia juega en el desarrollo de las conductas antisociales en los adolescentes. Es por ello que nuestro objetivo es determinar la influencia conjunta de diversas variables familiares, tanto estructurales como funcionales, en la prevalencia de la conducta antisocial adolescente de una población en general. Se parte de la hipótesis de que un funcionamiento familiar positivo se constituye como un factor protector frente a las conductas antisociales; mientras que variables como: consumo de alcohol en los padres, ausencia de alguno de ellos, problemas económicos, violencia de pareja y maltrato hacia los hijos se constituirán como un factor de riesgo de dichas conductas.

\section{Método}

\section{Participantes}

La muestra de este estudio estuvo constituida por 929 alumnos que cursan el $3^{\circ}, 4^{\circ}$ y $5^{\circ}$ grado de educación secundaria en 15 instituciones educativas tanto públicas como privadas de Arequipa metropolitana. De ellos, 437 son mujeres $(47 \%)$ y 492 son varones $(53 \%)$, cuyas edades fluctúan entre los 13 y 17 años, con una edad promedio de 15.35 años.

Los colegios fueron seleccionados teniendo en cuenta los parámetros de la Asociación Peruana de Empresas de Investigación de Mercados (APEIM, 2014), la cual anualmente permite tener información sobre los niveles socioeconómicos (NSE) de todo el Perú, por departamentos y áreas de residencia. Para el presente estudio, se siguió la distribución de los NSE de Arequipa metropolitana (AB, C, D y E) y después se seleccionaron a los colegios representativos de cada estrato.

\section{Procedimiento}

En primer lugar, se contactó con los centros educativos a través de sus directores mediando oficios y entrevistas en las cuales se les explicó los objetivos, importancia y procedimientos de la investigación. Luego de la aceptación de las autoridades se coordinaron las fechas y los salones de $3^{\circ}$ a $5^{\circ}$ en los que se iba a evaluar, en cada colegio. La evaluación tuvo lugar en dos fechas, con una duración aproximada de 25 y 20 minutos, respectivamente. El equipo de investigación explicó a los alumnos en qué consistía la evaluación y que su participación seria voluntaria además de anónima (se utilizaron códigos numéricos en vez del nombre de los alumnos).

\section{Variables e instrumentos}

Variables demográficas y socioeconómicas. Incluimos preguntas que indagan sobre la estructura familiar: la cantidad de hermanos, edad de los padres, situación laboral de los padres, estado civil de los padres, el tipo de familia (nuclear, monoparental o reconstruida); además de conocer sobre la existencia de problemas económicos, consumo de alcohol en los padres y violencia entre padres. Se utilizó también, la Evaluación del nivel socioeconómico (Vera-Romero \& Vera-Romero, 2013) para determinar en qué NSE se encontraban los alumnos, ya que si bien se escogieron los colegios siguiendo los criterios del APEIM, en una misma clase pueden coexistir estudiantes de NSE diferentes.

Relación familiar. Para la evaluación de esta variable, utilizamos la dimensión Relación de la Escala de clima social familiar (FES) cuyos autores son Moos y Trickett, la cual ha sido validada y estandarizada en Lima (Ruiz \& Guerra, 1993). La dimensión Relación mide las relaciones internas exigidas para un buen clima en la familia; para ello intervienen las sub-escalas de Cohesión, Expresividad e Inexistencia de Conflicto. 
Los coeficientes de consistencia interna en el presente estudio son: .75 para cohesión, .60 para expresividad y .65 para conflicto.

Cohesión y adaptabilidad familiar. Estas variables fueron tomadas de la Escala de Evaluación del Sistema Familiar (FACES III), cuyos autores son Olson, Portner y Lavee y la cual ha sido aplicada en diversos estudios a nivel nacional (Pinto, et al., 2004; Tueros, 2004). Presenta 20 ítems, con un tiempo aproximado de aplicación de 10 minutos. Tiene dos dimensiones: Cohesión, que mide cuán unidos se encuentran los miembros de la familia y la dimensión Adaptabilidad que evalúa el grado de estabilidad de la familia ante diversas situaciones. La fiabilidad de la sub-escala de cohesión es $\alpha=.83$ y $\alpha=$ .68 para la escala de adaptabilidad.

Satisfacción familiar. Esta variable fue medida a través de Escala de Satisfacción Familiar (CSF) de Olson y Wilson, en su versión validada para el Perú por Bueno (1996). Tiene 14 ítems, con un tiempo aproximado de aplicación de 6 minutos. La escala mide el grado en que los adolescentes están conformes con las normas y la dinámica familiar. Tiene dos dimensiones: satisfacción con la Cohesión y con la Adaptabilidad. En el presente estudio, la fiabilidad de la escala global según es de $\alpha=$ .87 , mientras que la fiabilidad para cada una de las subescalas es de $\alpha=.80$ y $\alpha=.77$, respectivamente.

Comunicación entre padres e hijos. Para su medición utilizamos el Cuestionario de Comunicación Familiar de Barnes y Olson, el cual tiene una versión validada en el Perú (Bueno, 1996). Esta escala está compuesta por 20 ítems y dos sub-escalas que evalúan, la comunicación con la madre y la comunicación con el padre. Las respuestas a los ítems varían de 1 (nunca) a 5 (siempre). Ambas sub-escalas presentan una estructura de dos factores que se refieren al grado de apertura y presencia de problemas en la comunicación familiar. La fiabilidad de la escala en esta investigación es en el factor apertura tanto de la madre como del padre es de $\alpha=.87$; mientras que es de $\alpha=.68$ en problemas en la comunicación con la madre y de $\alpha=$ .66 con el padre. Para realizar el análisis de ecuaciones estructurales, estos cuatro factores se fusionaron en uno solo denominado comunicación familiar. Para ello, invertimos las puntuaciones de los dos factores de problemas en la comunicación para luego, sumarlos con los otros dos factores de apertura en la comunicación, procedimiento realizado en otras investigaciones similares (Cava, Musitu \& Murgui, 2006).

Conductas antisociales. Se administró la sub-escala de conductas antisociales de la Escala de Conductas Antisociales y Delictivas [A-D] de Seis dedos, en su versión validada y adaptada en Lima (Ponce, 2003). La sub-escala de Conductas Antisociales tiene 20 ítems; la tarea consiste en leer las frases e informar si se han realizado las conductas que describen las frases, utiliza un formato de respuesta dicotómica (sí o no). Ponce (2003) reportó un coeficiente alfa de .87 y en esta investigación el coeficiente es de .86 para dicha sub-escala.

\section{Análisis de datos}

Se calcularon estadísticos univariados como las frecuencias de las variables categóricas, las medias y las desviaciones estándar de las variables cuantitativas y los coeficientes Alfa de Cronbach de las diversas escalas empleadas. Las medias de dichas escalas constituyen los índices de las variables a relacionar, con dichos índices se elaboró una matriz de correlaciones para probar el modelo propuesto. Además, se comparó por medio de ANOVA y t de Student, las diferencias en la conducta antisocial entre diversas variables demográficas contempladas en este estudio para incluirlas en el modelo, todo ello mediante el software IBM SPSS 20.

Generamos un modelo de ecuaciones estructurales mediante el módulo SPSS AMOS 22 (Arbuckle, 2013). Este modelo contempla dos pasos: el modelo de medición, que consiste en un análisis factorial confirmatorio y que une variables observadas correlacionadas entre sí para dar lugar a una variable latente o factores. En este estudio se hipotetizó la presencia de dos factores: el de maltrato hacia los hijos, formado por el maltrato propinado por los padres y madres a los adolescentes evaluados; mientras que el factor de funcionamiento familiar estuvo conformado por las relaciones familiares, la cohesión, adaptabilidad, satisfacción familiar y la comunicación entre padres e 
hijos. Por otro lado, el modelo estructural se refiere a las relaciones entre las variables latentes o factores obtenidos en el modelo de medición con las variables observadas (Kline, 2011).

Para determinar si los datos respaldan el modelo teórico, se utilizan diversos índices de bondad de ajuste: el estadístico Chi-cuadrado $\left(\chi^{2}\right)$ comparándolo con sus grados de libertad; el Índice de Ajuste Normalizado NFI, que contrasta el modelo propuesto con el modelo nulo; el Índice de Ajuste Comparativo CFI, el cual mide la mejora en la medición de la no centralidad de un modelo; el Índice de Bondad de Ajuste GFI, que es un índice de variabilidad y representa el grado general de ajuste al modelo; además del Índice del Error de Aproximación Cuadrático Medio RMSEA, que evidencia el ajuste que se puede anticipar con el valor total de la población y no con el de la muestra. Un modelo propuesto presenta un buen ajuste a los datos observados cuando el producto entre el Chi-cuadrado y los grados de libertad es menor a tres, los índices de ajuste NFI, CFI, y GFI son iguales o superiores a .90 y el RMSEA es menor a .08 (Byrne, 2010).

\section{Resultados}

Las correlaciones entre las variables de estudio, así como el análisis descriptivo de dichas variables según el sexo de los evaluados, se pueden apreciar en la Tabla 1. La mayoría de dichas correlaciones son significativas y en la dirección esperada, aunque de baja intensidad en lo que respecta a las conductas antisociales.

Respecto a las variables demográficas incluidas en el estudio, el ANOVA realizado muestra que existen diferencias significativas en las conductas antisociales según el estado civil de los padres $\left(F_{(2,926)}=3.924, p=\right.$ $.020)$, consumo de alcohol por parte de los padres $\left(F_{\beta}\right.$, $\left.{ }_{925}=6.938, p<.001\right)$, violencia entre los padres $\left(F_{\beta,}\right.$ $\left.{ }_{925}=2.826, p=.038\right)$ y maltrato por parte de la madre $\left(F_{(3,925)}=8.261, p<.001\right)$ o padre $\left(F_{(3,925)}=5.752, p=\right.$ .001) hacia los hijos. En cambio no se encontraron diferencias en cuanto al tipo de familia $\left(F_{\beta, 924)}=1.229, p\right.$ $=.298)$ y los problemas económicos familiares $\left(F_{(4,924)}=\right.$ $1.595, p=.173)$. Por otro lado, hallamos diferencias en las conductas antisociales teniendo en cuenta la situación laboral de la madre $\left(t_{011)}=-3.557, p<.001\right)$; pero para el caso de la situación laboral paterna no hay diferencias significativas $\left(t_{(898)}=-0.747 ; p=.455\right)$. Además contrastamos las conductas antisociales según el sexo de los adolescentes $\left(t_{(927)}=3.611, p<.001, d\right.$ Cohen $=$ .24) y encontrando que los varones cometen con más conductas antisociales que las mujeres. Un análisis ítem por ítem muestra que los varones tienden a: alborotar $\left(t_{(927)}=-3.646, p<.001\right)$, entrar en lugares prohibidos $\left(t_{(927)}=-2.994, p=.003\right)$, decir groserías $\left(t_{(927)}=-3.406, p\right.$ $=.001)$, hacer bromas pesadas $\left(t_{(027)}=-4.457, p<.001\right)$, enfrentarse a figuras de autoridad $\left(t_{(927)}=-2.747, p=.006\right)$ y pelearse con otros $\left(t_{(927)}=-6.305, p<.001\right)$; mientras que las mujeres tienden a llegar tarde al colegio o alguna reunión $\left(t_{927)}=1.995, p=.046\right)$.

Calculamos el modelo de medición en la muestra total que incluye a dos factores latentes: el maltrato hacia los hijos, compuesto por el maltrato de la madre con un peso factorial de $.90 \mathrm{y}$ del padre con un peso de .45. El funcionamiento familiar quedó conformado por la Cohesión, con un peso factorial de .78; la Adaptabilidad, con .38; la relación, con .75; satisfacción familiar, con .79 y comunicación familiar, con un peso de .64. Todos los pesos factoriales fueron muy significativos $(p<.001)$. Con estos datos se generó un modelo estructural inicial que se puso a prueba en varones y mujeres por separado, teniendo en cuenta las diferencias encontradas en las conductas antisociales.

Sin embargo, al ser puesto a prueba, los índices de bondad de ajuste no fueron los adecuados ni en mujeres ni en varones (ver figura 1); por lo que, teniendo en cuenta estos resultados, nos vimos en la necesidad de reespecificar el modelo en ambos sexos por separado, teniendo en cuenta el nivel de significación de los parámetros (relaciones entre variables).

Luego de reespecificar el modelo inicial, los índices de ajuste para el modelo final de las mujeres fueron adecuados (ver Figura 2). De acuerdo con este modelo, un adecuado funcionamiento familiar caracterizado por miembros de la familia satisfechos, que mantienen buenas relaciones entre sí, que se hallan unidos, se adaptan a los cambios que se van presentando y tienen una buena comunicación - ejerce 
Tabla 1

Correlaciones de Pearson y estadísticos descriptivos de las variables de estudio

\begin{tabular}{lccccccc}
\hline & 1 & 2 & 3 & 4 & 5 & 6 & 7 \\
\hline 1. Conductas antisociales & 1 & $-.127^{* *}$ & $-.177^{* *}$ & $-.189^{* *}$ & $-.234^{* *}$ & $-.086^{* *}$ & $-.228^{* *}$ \\
2. Número de hermanos & & 1 & -.012 & -.012 & .014 & -.028 & .018 \\
$\begin{array}{l}\text { 3. Relación } \\
\text { 4. Satisfacción familiar }\end{array}$ & & & 1 & $.616^{* *}$ & $.559^{* *}$ & $.233^{* *}$ & $.478^{* *}$ \\
5. Cohesión & & & & 1 & $.614^{* *}$ & $.354^{* *}$ & $.454^{* *}$ \\
6. Adaptabilidad & & & & 1 & $.473^{* *}$ & $.553^{* *}$ \\
$\begin{array}{l}\text { 7. Comunicación familiar } \\
\text { Media varones/ }\end{array}$ & & & & & & 1 & $.227^{* *}$ \\
$\quad$ mujeres & $5.62 /$ & $2.32 /$ & $18.0 /$ & $50.08 /$ & $37.15 /$ & $30.21 /$ & $137.51 /$ \\
\hline $\begin{array}{l}\text { D.E. varones/ } \\
\text { mujeres }\end{array}$ & 4.57 & 2.71 & 17.34 & 49.02 & 36.95 & 28.69 & 136.37 \\
\hline
\end{tabular}

$* * p<0.01$

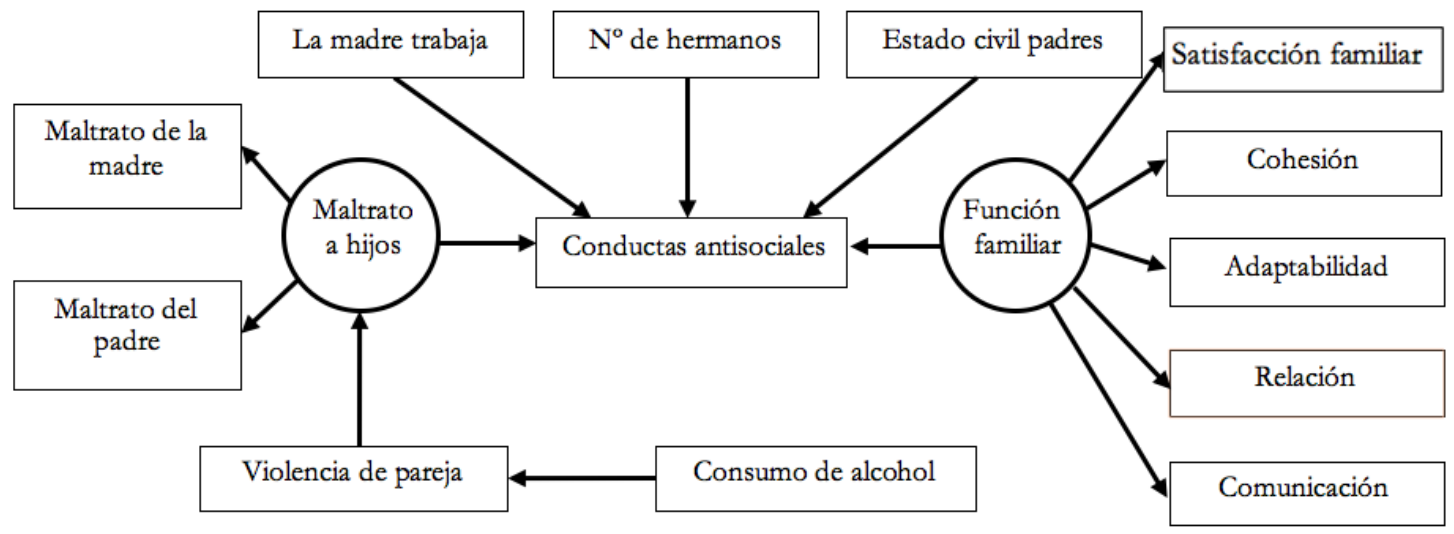

Figura 1. Modelo estructural inicial de la influencia de la familia sobre las conductas antisociales de los adolescentes para ambos sexos.

Nota. En las mujeres: $\chi^{2}=247.87, p<.001 ; \chi^{2} / \mathrm{gl}=3.922$; $\mathrm{NFI}=.79 ; \mathrm{GFI}=.81 ; \mathrm{CFI}=.83$; RMSEA $=.82$. En los varones: $\chi^{2}=279.17, p<.001 ; \chi^{2} / \mathrm{gl}=4.43 ; \mathrm{NFI}=.77 ; \mathrm{GFI}=.79 ; \mathrm{CFI}=.81$ y $\mathrm{RMSEA}=.84$. 
un efecto protector frente a las conductas antisociales de las hijas adolescentes $(\lambda=-.29, p<.001)$. Por el contrario, una familia en la que existe el maltrato hacia las hijas por parte de los padres, principalmente de la madre, incrementa la probabilidad de que las adolescentes presenten conductas antisociales como respuesta o desfogue a esta situación $(\lambda=.26, p<.001)$. Otro punto importante a considerar es que el maltrato hacia las hijas se ve influido, a su vez, por la violencia de pareja entre los padres $(\lambda=.26, p<.001)$ y el consumo de alcohol por parte de alguno de sus progenitores $(\lambda=.27, p<.001)$.

Para el caso del modelo reespecificado en los varones (ver Figura 3), encontramos que al igual que en el modelo estructural de las mujeres, un adecuado funcionamiento familiar genera un efecto protector

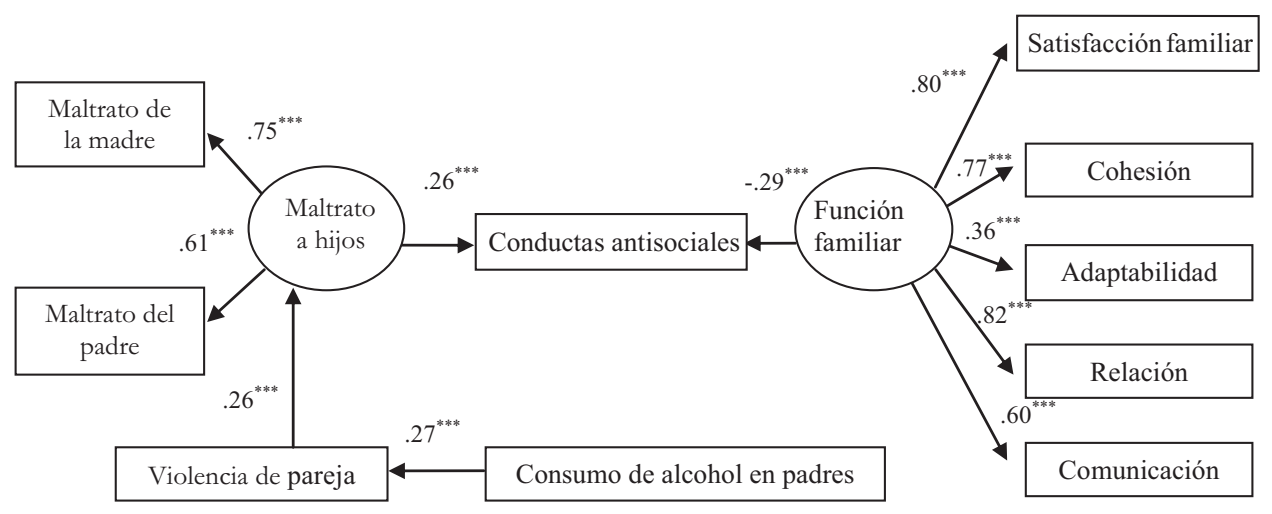

Figura 2. Modelo estructural final de la influencia de la familia sobre las conductas antisociales en las adolescentes mujeres Nota. $\chi^{2}=166.061 ; p<0.001 ; \chi^{2} / \mathrm{gl}=3.34 ; \mathrm{NFI}=.90 ; \mathrm{GFI}=.90 ; \mathrm{CFI}=0.92 ; \mathrm{RMSEA}=.079 ; \mathrm{R}^{2}$ (Conductas antisociales) $=.149$ $* * * p<.001$

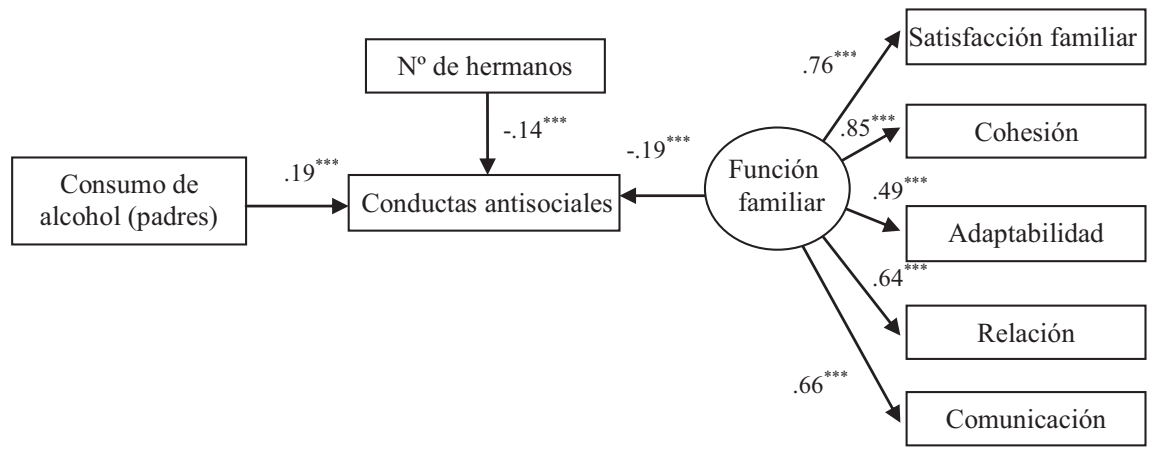

Figura 3. Modelo estructural final de la influencia de la familia sobre las conductas antisociales en los adolescentes varones Nota. $\chi^{2}=78.57, p<.001 ; \chi^{2} / \mathrm{gl}=3.05 ; \mathrm{NFI}=.91 ; \mathrm{GFI}=.92 ; \mathrm{CFI}=.93 ; \mathrm{RMSEA}=.077 ; \mathrm{R}^{2}$ (Conductas antisociales) $=.10$ 
contra las conductas antisociales en los adolescentes varones $(\lambda=-.19 ; p<.001)$, así como tener una mayor cantidad de hermanos $(\lambda=-.14 ; p<.001)$. Por otro lado, el consumo de alcohol por parte de alguno o ambos padres incrementa la probabilidad de conductas antisociales en los hijos $(\lambda=.19 ; p<.001)$.

\section{Discusión}

Los resultados ponen de manifiesto la existencia de diferencias significativas entre los adolescentes varones y mujeres. De manera específica, los hallazgos del presente estudio indican que en Arequipalos adolescentes varones presentan más conductas antisociales que la mujeres, lo que es concordante con estudios previos (Jiménez, et al., 2014; Martínez \& Gras, 2007; Quiroz del Valle, et al., 2007; Secretaría Nacional de la Juventud - MINEDU, 2013). Esto, en general, induce a pensar que los hombres, comparados con las mujeres, están en mayor riesgo de realizar actos antisociales y tienen mayor tendencia a involucrarse en actos delictivos.

En la presente investigación, un mayor número de varones, en comparación con las mujeres, admitió haber realizado conductas agresivas, como pelearse con otros (con golpes, insultos o palabras ofensivas), contestar a figuras de autoridad o decir groserías; más mujeres, por su parte, indicaron llegar tarde al colegio o reunión. Estos resultados concuerdan con los hallazgos de otros investigadores (Bellido, et al., 2013; Gaeta \& Galvanovskis, 2011), en el sentido de que los varones tienden a ser más agresivos en sus conductas que las mujeres.

Los resultados hallados respecto a la estructura familiar muestran que una mayor cantidad de hermanos influye en la aparición de las conductas antisociales en adolescentes varones, mientras que en el caso de adolescentes mujeres no se observó influencia alguna. El resultado obtenido corroboraría lo hallado por Sobral et al. (2000). Si bien es cierto, la mayoría de investigaciones refiere que una mayor cantidad de hermanos incrementa la conducta antisocial (Antolín, 2011; Torrente \& Rodríguez, 2004), se hace necesario mencionar a Romero et al. (2000), quienes argumentan que más que el número de hermanos, una relación tensa entre ellos ejerce una influencia más fuerte en las conductas antisociales.

Otras variables de la estructura familiar como el tipo de familia (nuclear, uniparental, reconstruida) o estado civil de los padres, no muestran relación con la conducta antisocial de los adolescentes, lo cual discrepa con lo hallado por otros investigadores que indican que, principalmente, la separación de los padres está relacionada con diversas manifestaciones clínicas en los hijos, entre ellas, las conductas agresivas (Torrente \& Rodríguez, 2004). Al respecto y siguiendo lo planteado por Antolín y Oliva (2009), esto se puede explicar por el hecho de que en esta investigación se evaluó una muestra de la población general de adolescentes; mientras que la mayoría de los estudios realizados respecto a la influencia de la estructura familiar en las conductas antisociales se han dado en poblaciones institucionalizas; es así que, nuestros datos tienden al promedio y no son tan extremos, como consecuencia de ello no se produce asociación entre las variables. Otro punto importante es que, a lo largo de diversas investigaciones, se ha ido constando que el efecto de las variables estructurales es más bien indirecto, mediado por las variables de funcionabilidad familiar (Hoffman, 1993 citado por Luengo, Romero, Gómez-Fragüela, Guerra, \& Lence, 1998)

$\mathrm{Al}$ respecto, las variables de funcionabilidad familiar demostraron influir en las conductas antisociales de los adolescentes. Respecto a los factores protectores en ambos sexos encontramos que un adecuado funcionamiento familiar - relaciones estables, miembros unidos que se adaptan a los cambios que se presenten, que se encuentran satisfechos con su familia y que tienen una comunicación fluida entre padres e hijos - disminuye la probabilidad de presentar conductas antisociales. Esto congruente con lo encontrado por diversos investigadores que señalan la importancia de un buen clima familiar (Matalinares, et al., 2010), niveles de cohesión y adaptabilidad que aseguren una correcta funcionabilidad de la familia que promueva el apego (Jiménez, Musitu, \& Murgui, 2006; H. Rodríguez, Espinoza, \& Pardo, 
2013; J. Rodríguez, 2011), una buena satisfacción familiar (Araujo, 2005; Ponce, 2003) y una adecuada comunicación familiar (Estévez, et al., 2007) para que los hijos se desarrollen adecuadamente y no presenten problemas en la adolescencia.

Por otro lado, entre los factores de riesgo ligados a la funcionabilidad familiar, hallamos que tanto para las mujeres como para los varones, el consumo de alcohol por parte de los progenitores constituye un factor de riesgo de conducta antisocial, lo cual está ampliamente documentado en diversas investigaciones, ya que fomenta la violencia familiar tanto hacia la pareja como hacia los hijos, además de una serie de problemas agregados entre los que resalta la conducta antisocial en los adolescentes (Frías, López, \& Díaz., 2003; Musitu, Estévez, Jiménez, \& Herrero, 2007). Para el caso particular de las mujeres, el maltrato físico y verbal como estilo disciplinario o de control parental, aumenta la probabilidad de que cometan conductas antisociales, lo que confirmaría lo sustentado por la teoría (Belsky, 2005; Bronfenbrenner, 1985, 1987) y lo hallado en diversas investigaciones (Frías, López, \& Diaz, 2003; Rodríguez, 2011).

Teniendo en cuenta las diferencias encontradas en la presentación de las conductas antisociales y la influencia de los factores familiares entre varones y mujeres, es necesario que en futuras investigaciones se tomen en cuenta las variables individuales que han demostrado funcionar como mediadoras o catalizadoras entre los factores familiares y la conducta antisocial, las cuales pueden reducir o incrementar su influencia como son: personalidad, impulsividad o búsqueda de sensaciones (Sobral, et al., 2000), autoestima, empatía, actitudes hacia la agresión o problemas psicopatológicos (Garaigordobil, 2005; Kazdin, 1993). Otras variables a considerar son las ligadas al ambiente escolar y social en el que vive el individuo y que se combinan con la influencia de la familia para generar o no conductas antisociales en los adolescentes (Bronfenbrenner, 1985, 1987; Rodríguez, 2011; Romero, et al., 2000). También es notoria la necesidad de realizar una línea de investigación en nuestro país sobre la génesis de las conductas antisociales y su relación con diversos problemas socio-emocionales.
Además, para generar programas preventivos o de rehabilitación que disminuyan las conductas antisociales en los adolescentes, es necesario detectar la propensión y riesgo de presentar este problema, considerando que cada persona experimenta un grado diferente de vulnerabilidad hacia las conductas antisociales y delictivas. Esto sin olvidar que en el mundo real las diversas variables operan de modo interrelacionado, interdependiente e incluso de forma simultánea sirviendo como factores de riesgo o protección frente a las conductas antisociales (Sobral, et al., 2000; Morales, 2013).

\section{Referencias}

Antolín, L. (2011). La conducta antisocial en la adolescencia. Una aproximación ecológica. (Tesis doctoral). Recuperada de: http://fondosdigitales.us.es/tesis/tesis/1519/ la-conducta-antisocial-en-la-adolescencia-unaaproximacion-ecologica/

Antolín, L., \& Oliva, A. (2009). Contexto familiar y conducta antisocial infantil. Anuario de Psicología, 40(3), 313-327.

Asociación Peruana de Empresas de Investigación de Mercados. (2014). Niveles socioeconómicos 2014. Recuperado 20 de setiembre del 2014 de http:// www.apeim.com.pe/wp-content/themes/apeim/ docs/nse/APEIM-NSE-2014.pdf

Araujo, E. (2005). La Satisfacción Familiar y su relación con la Agresividad y las Estrategias de Afrontamiento en Adolescentes de Lima Metropolitana. Cultura, 19, 13-38.

Arbuckle, J. (2013). IBM SPSS Amos 21 User's Guide: IBM.

Belsky, J. (2005). Family influences on psychological development. Psychiatry, 4(7), 41-44. doi: 10.1016 / j.mppsy.2008.05.006

Bellido, F., Rivera, R., Salas, J. C., Bellido, V., Peña, N., Villasante, G., \& Casapia, Y. (octubre, 2013). Actitudes hacia el Bullying y sus diversas manifestaciones en escolares de educación secundaria en Arequipa Metropolitana. Trabajo presentado en XVI Congreso Peruano y VI Congreso Internacional de Psicología, Arequipa. 
Benavides, M., \& León, J. (2013). Una mirada a la violencia física contra los niños y niñas en los hogares peruanos: Magnitudes, factores asociados y transmisión de la violencia de madres a hijos e hijas. Lima: Grupo de Análisis para el Desarrollo GRADE.

Bronfenbrenner, U. (1985). Contextos de crianza del niño. Problemas y prospectiva. Infancia y aprendizaje, 29, 45-55.

Bronfenbrenner, U. (1987). La ecología del desarrollo bumano. Madrid: Paidós.

Bueno, R. (1996). Validación, confiabilidad y correlación entre las escalas de comunicación padres-adolescentes y satisfacción familiar en estudiantes de una universidad de Lima. (Tesis de maestria inedita). Universidad San Martín de Porres, Lima-Perú.

Byrne, B. (2010). Structural equation modeling with AMOS (2 ed.). New York: Routledge, Taylor \& Francis Group.

Carpio de La Torre, R., \& Alay, D. (2012). Modelo ecológico aplicado al estudio de la conducta antisocial. Rev. Psicología Arequipa, 2(2), 167-179.

Cava, M. J., Musitu, G., \& Murgui, S. (2006). Familia y violencia escolar: el rol mediador de la autoestima y la actitud hacia la autoridad institucional. Psicothema, 18(3), 367-373

CEPAL. (2008). Panorama Social 2008. Santiago de Chile: Organización de las Naciones Unidas.

Cuevas del Real, M. C. (2004). Los factores de riesgo y la prevención de la conducta antisocial. En A. Silva (Ed.), Conductas antisociales: un enfoque psicológico (Vol. I, pp. 372). México, D.F.: Pax, México.

Estévez, E., Murgui, S., Moreno, D., \& Musitu, G. (2007). Estilos de comunicación familiar, actitud hacia la autoridad institucional y conducta violenta del adolescente en la escuela. Psicothema, 19(1), 108-113.

Florenzano, R., Valdés, M., Cáceres, E., Casassus, M., Sandoval, A., Santander, S., \& Cálderón, S. (2009). Percepción de la relación parental entre adolescentes mayores y menores de 15 años. Rev Chil Pediatr, 80(6), 520-527.
Frías, M., López, A., \& Díaz, S. (2003). Predictores de la conducta antisocial juvenil: un modelo ecológico. Estudios de Psicología, 8(1), 15-24.

Frías, M., Rodríguez, I., \& Gaxiola, J. C. (2003). Efectos conductuales y sociales de la violencia familiar en niños mexicanos. Revista de Psicología de la PUCP, 21(1), 42-69.

Gaeta, M., \& Galvanovskis, A. (2011). Propensión a conductas antisociales y delictivas en adolescentes mexicanos. Psicología Iberoamericana 19(2), 47-54.

Garaigordobil, M. (2005). Conducta antisocial durante la adolescencia: correlatos socio-emocionales, predictores y diferencias de género. Psicología Conductual, 13(2), 197-215.

Gaxiola, J., \& Frías, M. (2008). Un modelo ecológico de factores protectores del abuso infantil: un estudio con madres mexicanas. Medio Ambiente y Comportamiento Humano, 9, 13-31.

Gracia, E., Fuentes, M., \& García, F. (2010). Barrios en Riesgo, Estilos de Socialización Parental y Problemas de Conducta en Adolescentes. Intervención Psicosocial, 19(3), 265-278.

Herrera, D., \& Morales, H. (2005). Comportamiento antisocial durante la adolescencia teoría, investigación y programas de prevención. Revista de Psicología de la PUCP, 23(2), 203-247.

Jiménez, T., Estévez, E., \& Murgui, S. (2014). Ambiente comunitario y actitud hacia la autoridad: relaciones con la calidad de las relaciones familiares y con la agresión hacia los iguales en adolescentes. Anales de Psicología, 30(3), 1086-1095. doi: 10.6018 / analesps.30.3.160041

Jiménez, T., Musitu, G., \& Murgui, S. (2006). Funcionamiento y comunicación familiar y consumo de sustancias en la adolescencia: el rol mediador del apoyo social. Revista de Psicología Social, 21(1), 21-34.

Kazdin, A. (1993). Tratamientos conductuales y cognitivos de la conducta antisocial en niños: avances de la investigación. Psicología Conductual, 1(1), 111-144.

Kline, R. (2011). Principles and practice of structural equation modeling (3 ed.). New York: The Guilford Press. 
Larrain, S. (2008). Maltrato infantil y relaciones familiares en Chile. Análisis comparativo. 1994-20002006. Revista Cbilena Pediatrica, 79(1), 68-79.

López, K., \& da Costa, M. (2008). Conducta antisocial y consumo de alchol en adolescentes escolares. Revista Latinoamericana de Enfermedades Mentales, 16(2), 1-7.

Luengo, M. A., Romero, E., Gómez-Fragüela, J., Guerra, A., \& Lence, M. (1998). La prevención del consumo de drogas y la conducta antisocial en la escuela: análisis y evaluación de un programa. Madrid: Universidad de Santiago de Compostela.

Martínez, G., \& Gras, M. (2007). La conducta antisocial percibida por adolescentes de Enseñanza Secundaria Obligatoria: frecuencia, contexto y atribución causal. Apuntes de Psicología, 25(3), 285-304.

Martínez, M., Robles, C., Utria, L., \& Amar, J. (2014). Legitimación de la violencia en la infancia un abordaje desde el enfoque ecológico de Bronfenbrenner. Psicología desde el Caribe, 31(1), 133-160. doi: 10.14482/ psdc.31.1.4930

Matalinares, M., Arenas, C., Sotelo, L., Diaz, G., Dioses, A., Yaringaño, J... Tipacti, R. (2010). Clima familiar y agresividad en estudiantes de secundaria de Lima metropolitana. Revista IIPSI, 13(1), 109-128.

Mirella, F. (2011). El costo económico de la delincuencia organizada en el Perú: estudio sobre los costos directos que el crimen ocasiona al estado y las familias. Lima: Oficina de las Naciones Unidas contra la Droga y el Delito.

Morales, H. (2008). Factores Asociados y Trayectorias del Desarrollo del Comportamiento antisocial durante la Adolescencia: Implicancias para la Prevención de la Violencia Juvenil en America Latina. Revista Interamericana de Psicología, 42(1), 129-142.

Morales, H. (2013). Comportamiento antisocial persistente y limitado a la adolescencia entre infractores institucionalizados (Tesis de licenciatura). Pontifica Universidad Católica del Perú, Lima.

Musitu, G., Estévez, E., Jiménez, T., \& Herrero, J. (2007). Familia y conducta delictiva y violenta en la adolescencia In S. Yubero, A. Blanco \& E. Larrañaga
(Eds.), Convivir con la violencia: un análisis desde la psicología y la educación de la violencia en nuestra ciudad. (pp. 135-150). Cuenca: Ediciones de la Universidad de Castilla-La Mancha.

Nagin, D., Farrington, D., \& Moffitt, T. (1995). Lifecourse trajectories of different types of offenders. Criminology, 33 (1),11-139.

Noroño, N., Cruz, R., Cadalso, R., \& Fernández, O. (2002). Influencia del medio familiar en niños con conductas agresivas. Revista Cubana de Pediatría, 74(2), 138-144.

Papalia, D., Wendkos, S., \& Duskin, R. (2005). Desarrollo Humano. México DF: Mc Graw Hill.

Peña, M., \& Graña, J. (2006). Agresión y conducta antisocial en la adolescencia: una integración conceptual. Psicopatología Clínica, Legaly Forense, 6, 9-23.

Pinto, F., Livia, J., Zegarra, V., Ortiz, M., Camacho, A., Isidoro, J., \& Martil, A. (2004). Trastornos de conducta y funcionamiento familiar en adolescentes. Revista Cientifica de Investigación en Psicología, 1, 55-66.

Ponce, C. (2003). Conductas antisocialesdelictivas y satisfacción familiar en grupos de estudiantes de quinto de secundaria de Lima metropolitana pertenecientes a diferentes estratos socioeconómicos. Revista de Investigación en Psicologia, 6(1), 104-125.

Quiroz del Valle, N., Villatoro, J., Juárez, F., Gutiérrez, M., Amador, N., \& Medina-Mora, M. (2007). La familia y el maltrato como factores de riesgo de conducta antisocial. Salud Mental, 30(4), 47-54.

Rodríguez, A., \& Torrente, G. (2003). Interacción familiar y conducta antisocial. Boletin de Psicología, 78, 7-19.

Rodríguez, H., Espinoza, A., \& Pardo, C. (2013). Función familiar y conductas antisociales y delictivas de adolescentes de instituciones públicas educativas de la ciudad de Ibagué-Colombia. Revista Vanguardia Psicológica, 3(2), 137-149.

Rodríguez, J. (2011). Conducta antisocial en grupo: una aproximación mediante ecuaciones estructurales. Revista Cenipec, 30, 237-264. 
Romero, E., Luengo, M. A., \& Gómez-Fragüela, J. (2000). Factores psicosociales y delincuencia. Escritos de Psicología, 4, 78-91.

Ruiz, C., \& Guerra, E. (1993). Clima social familiar de adolescentes y su influencia en el rendimiento académico (Tesis de licenciatura inedita). Universidad Nacional Mayor de San Marcos, Lima.

Sanabria, A., \& Uribe, A. F. (2010). Factores psicosociales de riesgo asociados a conductas problemáticas en jóvenes infractores y no infractores. Revista Diversitas-Perspectivas en Psicologia, 6(2), 257-274.

Secretaría Nacional de la Juventud - MINEDU. (2013). Criminalidad y violencia juvenil en el Perú. Exploración en el contexto y orígenes del comportamiento trasgresor entre los jóvenes. Lima: Ministerio de Justicia y Derechos Humanos.

Sobral, J., Romero, E., Luengo, M. A., \& Marzona, J. (2000). Personalidad y conducta antisocial: amplificadores individuales de los efectos contextuales. Psicothema, 12(4), 661-670.

Torrente, G., \& Rodríguez, A. (2004). Características sociales y familiares vinculadas al desarrollo de la conducta delictiva en pre-adolescentes y adolescentes. Cuadernos de Trabajo Social, 17, 99-115.

Tueros, R. (2004). Cohesión y adaptabilidad familiar y su relación con el rendimiento académico (Tesis de maestría inédita). Universidad Nacional Mayor de San Marcos, Lima.

Vera-Romero, O., \& Vera-Romero, F. (2013). Evaluación del nivel socioeconómico: presentación de una escala adaptada en una población de Lambayeque. Rev. cuerpo méd. $H N A A A, 6(1), 41-45$.

Villareal, M. E. (2009). Un modelo estructural del consumo de drogas y conducta violenta en adolescentes escolarizados (Tesis doctoral). Recuperada de: www.biblioteca.cij. gob.mx/Archivos/Tesis.../modeloestructural2.pdf 
